\title{
Erratum to: The serum levels of soluble interleukin-2 receptor $\alpha$ and lactate dehydrogenase but not of B2-microglobulin correlate with selected clinico-pathological prognostic factors and response to therapy in childhood soft tissue sarcomas
}

\author{
Ewa Bien • Malgorzata Rapala • Malgorzata Krawczyk • \\ Anna Balcerska
}

Published online: 29 November 2009

(C) Springer-Verlag 2009

\section{Erratum to: J Cancer Res Clin Oncol DOI 10.1007/s00432-009-0661-x}

This article was unfortunately submitted for publication with errors in Abstract and Keywords. The correct version of Abstract and Keywords are given below.

\begin{abstract}
Purpose To establish the clinical utility of serum soluble IL-2 receptor (sIL-2R $\alpha$ ), lactate dehydrogenase (LDH) and B2-microglobulin (B2-M) as markers for diagnosis, prognosis and treatment monitoring in childhood soft tissue sarcomas (STS).

Methods The markers were measured prospectively before treatment, in complete remission (CR) during and after therapy and at relapse in 35 children with STS and in 50 healthy children (once).
\end{abstract}

The online version of the original article can be found under doi:10.1007/s00432-009-0661-x.

\section{E. Bien $(\bowtie) \cdot A$. Balcerska}

Department of Paediatrics, Haematology,

Oncology and Endocrinology, Medical University of Gdansk,

7 Debinki Street, 80-211 Gdansk, Poland

e-mail: ewabien1@wp.pl

M. Rapala

Department of Paediatric Surgery,

Marciniak Hospital, Wroclaw, Poland

M. Krawczyk

Medical Student Association, Department of Paediatric,

Haematology, Oncology and Endocrinology,

Medical University of Gdansk, Gdansk, Poland
Results Serum sIL-2R $\alpha$ and LDH correlated with age thus they were presented as multiplications of the upper normal ranges for age. Pre-treatment levels of sIL-2R $\alpha$ and $\mathrm{LDH}$ but not of B2-M exceeded significantly those of controls. Elevated sIL-2R $\alpha$ levels correlated with more advanced stages, poor-risk histology and poor response to chemotherapy, higher LDH with incomplete primary tumour resection and increased B2-M with poor-risk histology. Patients' age $>10$ years, male gender and unfavourable tumour localisation were not accompanied by the markers' elevation. None of the markers predicted EFS and OS. Good response to chemotherapy was paralleled by significant decline of sIL-2R $\alpha$ and LDH pre-treatment levels while relapse-by sIL-2R $\alpha$ and LDH increase to values similar to those at diagnosis. Monitoring of B2-M did not reflect the disease course.

Conclusions sIL-2R $\alpha$ and LDH were proven to be promising markers for diagnosis and treatment monitoring in children with STS. The markers correlated also with some important prognostic clinico-pathological factors for childhood STS; however, they failed to predict EFS and OS. Measurements of serum B2-M were shown to have no clinical value in the diagnostics, prognostics and treatment monitoring in paediatric STS.

Keywords Soft tissue sarcomas $\cdot$ Children $\cdot$ Serum soluble IL-2 receptor $\alpha$. Lactate dehydrogenase . B2-microglobulin . Tumour markers 